\title{
Factors associated with continued participation in a matched monetary incentive programme at local farmers' markets in low-income neighbourhoods in San Diego, California
}

\author{
Amanda R Ratigan ${ }^{1,2, *}$, Suzanne Lindsay ${ }^{1,3}$, Hector Lemus ${ }^{1}$, Christina D Chambers ${ }^{2}$ \\ Cheryl AM Anderson ${ }^{2}$, Terry A Cronan ${ }^{4}$, Deirdre K Browner ${ }^{5}$ and Wilma J Wooten ${ }^{5}$ \\ 'Graduate School of Public Health, San Diego State University, 5500 Campanile Drive, San Diego, CA 92182, \\ USA: ${ }^{2}$ Department of Family Medicine and Public Health, University of California San Diego, School of Medicine, \\ 9500 Gilman Drive, San Diego, CA 92093, USA: ${ }^{3}$ Institute of Public Health, San Diego State University, San Diego, \\ CA, USA: ${ }^{4}$ Department of Psychology, San Diego State University, San Diego, CA, USA: ${ }^{5}$ County of San Diego \\ Health and Human Services Agency, San Diego, CA, USA
}

Submitted 28 October 2016: Final revision received 21 May 2017: Accepted 1 June 2017: First published online 31 July 2017

\begin{abstract}
Objective: The Farmers' Market Fresh Fund Incentive Program is a policy, systems and environmental intervention to improve access to fresh produce for participants on governmental assistance in the USA. The current study examined factors associated with ongoing participation in this matched monetary incentive programme.

Design: Relationship of baseline factors with number of Fresh Fund visits was assessed using Poisson regression. Mixed-effects modelling was used to explore changes in consumption of fruits and vegetables and diet quality.

Setting: San Diego, California.

Subjects: Recipients of Supplemental Nutrition Assistance Program (SNAP), Special Supplemental Nutrition Program for Women, Infants, and Children (WIC) and Supplemental Security Income (SSI) who attended participating farmers' markets from 2010 to 2012 ( $n$ 7298).

Results: Among those with participation for $\leq 6$ months, factors associated with increased visits included reporting more daily servings of fruits and vegetables $(\mathrm{F} \& \mathrm{~V})$ at baseline, being Vietnamese or Asian/Pacific Islander, and eligibility because of SNAP/CalFresh or SSI ( $v$. WIC). Among those who came for 6-12 months, being Asian/Pacific Islander, eligibility because of SNAP/CalFresh and enrolling in the autumn, winter or spring were associated with a greater number of Fresh Fund visits. Among those who came for $>12$ months, being male and eligibility because of SSI were associated with a greater number of visits. Overall, the odds of increasing number of servings of F\&V consumed increased by $2 \%$ per month, and the odds of improved perception of diet quality increased by $10 \%$ per month.

Conclusions: Sustaining and increasing Fresh Fund-type programme operations should be a top priority for future policy decisions concerning farmers' market use in low-income neighbourhoods.
\end{abstract}

Current daily recommendations for fruit and vegetable (F\&V) intake in the USA range from five to thirteen servings depending on age, sex and activity level ${ }^{(1)}$; yet less than onethird of Americans report consuming two or more servings of fruits or three or more servings of vegetables per day ${ }^{(2)}$. Similar prevalence of low F\&V consumption has been noted in other high-income countries such as Canada, Australia and the $\mathrm{UK}^{(3-5)}$, as well as many low- and middle-income countries $^{(6)}$. Moreover, evidence suggests that consumption varies by individual and neighbourhood socio-economic status, where lower socio-economic status has been linked with decreased intake of F\&V in the USA ${ }^{(4,7-9)}$. Access to food retail stores with healthier products has been found to be associated with consumption of higher-quality foods ${ }^{(10-13)}$ and people who live in low-income and minority communities have decreased access to healthier food stores ${ }^{(11,14,15)}$. 
In recent years, farmers' markets have become increasingly common in higher-income countries. The US Department of Agriculture listed more than 8500 farmers' markets across the USA in 2017, while Australia had 200 markets, the UK had approximately 500 markets in 2013, and Canada had approximately 500 markets in $2009^{(16-19)}$. Furthermore, farmers' markets are becoming more popular in the USA as an approach for improving access to nutritious fresh foods for low-income consumers who receive government nutrition assistance. From 2008 to 2015 , there was a $761 \%$ increase in the number of authorized farmers' markets accepting Supplemental Nutrition Assistance Program (SNAP; also known as CalFresh in California) benefits through the use of electronic benefit transfer machines ${ }^{(20)}$. To further improve access and affordability, financial incentives to shop at farmers' markets have been implemented among economically disadvantaged populations who receive SNAP and Special Supplemental Nutrition Program for Women, Infants, and Children (WIC) nutrition benefits ${ }^{(21,22)}$.

Previously published data suggest that farmers' market use is associated with increased F\&V consumption among SNAP and WIC beneficiaries ${ }^{(23-25)}$; and furthermore, that farmers' market incentive programmes may increase spending of benefit money ${ }^{(26)}$, as well as the purchase and consumption of $F \& V^{(27-33)}$. As a result of this previous research, the San Diego County Health and Human Services Agency and the San Diego International Rescue Committee created a partnership in 2008 to fully implement the promising practice of financially incentivizing a primarily low-income refugee community to use government assistance monies and incentives to purchase fresh F\&V at the existing City Heights farmers' market. In 2010, the San Diego County Health and Human Services Agency used funding from the Centers for Disease Control and Prevention (CDC) to expand the Fresh Fund programme to four markets in low-income neighbourhoods and expanded to an academic-community practice partnership that included the Division of Child Development and Community Health at the University of California San Diego for content expertise, and the Institute for Public Health at San Diego State University for evaluation. Fresh Fund follows the CDC's leadership in shifting from a focus on individual health-risk behaviours to the implementation of policy, systems and environmental change for chronic disease prevention ${ }^{(34,35)}$. Policy decisions to allow farmers' markets to accept government assistance monies and to incentivize their participation are substantially different from attempts to convince individuals to change their eating habits.

While promising, researchers who have examined purchase and consumption patterns of F\&V by lowincome populations at farmers' markets lacked longitudinal or multivariate analyses ${ }^{(27-30)}$, did not control for potential confounding by participant characteristics ${ }^{(32)}$ or did not examine predictors of continued usage ${ }^{(33)}$.
In addition, many of the studies were short-term research projects and did not necessarily involve attempts to permanently embed the incentive programmes into the practice settings of existing farmers' markets as San Diego County attempted to do. Herman and colleagues reported the results of a 6-month nutritional intervention that assigned postpartum WIC participants to either an intervention group that received vouchers for shopping at farmers' markets or supermarkets, or a control group ${ }^{(31)}$. They found that the intervention group participants increased and sustained their consumption of $\mathrm{F} \& \mathrm{~V}$ for 6 months after the intervention ended.

Continued use of farmers' market incentive programmes may indicate a greater need. Therefore, a better understanding of the characteristics of individuals who continue to use farmers' market incentive programmes may help to develop more targeted advertising and outreach techniques to reach the low-income populations who need the assistance most. Therefore, the purpose of the current study was to examine the factors associated with the ongoing utilization of a farmers' market incentive programme among government nutrition assistance recipients in San Diego, California.

\section{Methods}

\section{Study population, study design and data collection} The evaluation period spanned from 1 June 2010 through 31 January 2012, during which time individuals were invited to participate in Fresh Fund at five farmers' markets in San Diego County if they received government assistance from SNAP, WIC or Supplemental Security Income (SSI/disability). Individuals younger than 18 years were eligible if they received disability income or were eligible for WIC because of pregnancy or having children under the age of 5 years. All 7298 Fresh Fund participants provided enrolment data, which included demographic characteristics, and were invited to complete voluntary, self-reported paper surveys at baseline and at 3-month intervals through the end of the evaluation period. Information collected in the surveys included diet, food purchasing behaviour and perceptions of the programme. The International Rescue Committee's Fresh Fund programme staff administered the surveys in person to participants who had limited literacy or those who spoke a language other than English (i.e. Spanish, Vietnamese, Chinese, Somali).

The Fresh Fund incentive consisted of 1:1 matching for each dollar exchanged to receive Fresh Fund tokens up to \$US 20 per month. An enrolment and exchange booth was permanently established at each of the participating markets to allow participants to enrol and then exchange public assistance money for tokens to be used to buy F\&V at the markets. Purchased and matched incentive tokens could be spent only at vendors who sold fresh produce or 
packaged foods, such as jams/spreads, breads, eggs, pasta, cheese and fish; however, tokens purchased using WIC funds could only be spent at vendors selling fresh produce. In addition, participants were not required to spend their tokens on the same day they were purchased. Records of market attendance were collected and maintained by trained Fresh Fund programme staff at each participating market and were used to determine the total number of Fresh Fund booth visits. In addition, the amount of government assistance or personal money participants exchanged to receive matched incentive tokens was also documented at each visit. Thus, programme staff collected information about visits and money exchanged each time the participant came to the market. More detailed survey data were collected only every 3 months. Participating farmers' markets were promoted through local outreach and media efforts by non-profit organizations, and included television and print campaigns, Fresh Fund mailers and flyers, and posters placed inside buses and bus shelters.

\section{Statistical analysis}

The first outcome of interest was the count of the total number of Fresh Fund visits (i.e. the number of times participants visited the Fresh Fund booth). The exposure variables that were examined were collected at baseline and included age, gender, race/ethnicity, number of people living in each household, source of government funding (WIC, SNAP/CalFresh, SSI), language in which the survey was conducted (English, Spanish, Vietnamese, Chinese, Somali), the farmers' market attended, season of Fresh Fund enrolment in 2010 or 2011 (spring: commencing 20 March; summer: commencing 21 June; autumn: commencing 22 September (23 September for 2011); winter: commencing 21 December), the amount of money exchanged to receive tokens (sum of personal cash/credit and government assistance money), and self-reported measures of daily consumption of $\mathrm{F} \& \mathrm{~V}(<1,1-2,3-4$ and $\geq 5$ servings/d), overall diet quality (very healthy, healthy, average, unhealthy, very unhealthy) and weekly spending on F\&V (<\$US 10, \$US 10-19, \$US 20-29, \$US 30-39, $\geq \$$ US 40).

Descriptive statistics were computed for all variables by the total months of Fresh Fund use (calculated as: (last visit date - enrolment date); categorized as $\leq 6$ months, 6-12 months and >12 months), including median and range for continuous variables, and frequency and percentage for categorical variables. The total number of Fresh Fund visits per visitor was not evenly distributed across months of Fresh Fund visits. For example, an individual could have visited fourteen times in 6 months, while another individual may have visited twice in 12 months. Presuming that the variables associated with number of visits may be different by the length of time the person participated in Fresh Fund, Poisson regression analyses were stratified by categorical months of Fresh Fund use ( $\leq 6$ months, 6-12 months and $>12$ months). The relationship of exposure variables to the number of Fresh Fund visits in each of the three categories of months of Fresh Fund use was analysed. Variables found to have significant bivariate associations with the number of visits at $P<0.20$ were included in a multivariate Poisson regression model using backward stepwise selection procedures. This level of significance was used to reduce the possibility of excluding potentially meaningful variables that may have occurred if a stricter cut-off value was used. Listwise deletion of missing variables was performed to restrict multivariate analysis to individuals with complete data. Overdispersion was assessed by examining the ratio of the deviance to the degrees of freedom for all Poisson regression models. Rate ratios with 95\% confidence intervals and $P$ values were reported to show the strength and direction of these associations. Tolerance values were calculated to assess collinearity among independent variables.

Additional analyses were conducted using linear regression to examine the relationship of government assistance and personal money exchanged with baseline daily consumption of $F \& V$ and the perception of overall diet quality. Multivariate models were adjusted for age, gender, race/ethnicity, source of government funding, enrolment market and season of enrolment. In addition, mixed-effects modelling with a random intercept was used to explore within-individual changes in the amount of money exchanged over the study period, and the average number of daily servings of F\&V and the perception of overall diet quality that was reported at baseline and at each follow-up assessment. Data were analysed using the statistical software packages IBM SPSS Statistics version 22 and SAS version 9.3; analyses were two-sided with $P<0.05$ considered to be statistically significant.

\section{Results}

A total of 7298 people enrolled in Fresh Fund from 1 June 2010 through 31 January 2012 at participating farmers' markets in San Diego County. Overall, the median age was 34 years (range: 7-100 years), 84.6\% were female, approximately half were Hispanic (49.5\%) and $56 \cdot 1 \%$ were eligible because of receiving WIC benefits (Table 1). More than half of participants (54.5\%) visited Fresh Fund once only (range: 1-36 visits), while the total length of Fresh Fund use ranged from 0 to 20 months. A total of 7017 participants completed a baseline survey at enrolment. Participants who were enrolled by October 2011 were eligible to complete a 3-6-month follow-up survey, and those who were enrolled by January 2011 were eligible to complete a 12-month survey. Among 1697 participants who returned multiple times for at least 3 months, 908 completed both a baseline and a 3-6-month follow-up 
Table 1 Characteristics of Fresh Fund participants, overall and according to length of Fresh Fund use. San Diego, California, 2010-2012 $(n 7298)^{*}$

\begin{tabular}{|c|c|c|c|c|c|c|c|c|c|}
\hline \multirow[b]{2}{*}{ Characteristic } & \multicolumn{2}{|c|}{ Total } & \multicolumn{2}{|c|}{$\leq 6$ months $(n 6127)$} & \multicolumn{2}{|c|}{ 6-12 months ( $n$ 692) } & \multicolumn{2}{|c|}{ >12 months ( $n$ 479) } & \multirow[b]{2}{*}{$P+$} \\
\hline & Median or $n$ & Range or $\%$ & Median or $n$ & Range or $\%$ & Median or $n$ & Range or $\%$ & Median or $n$ & Range or $\%$ & \\
\hline Age (years) ( $n$ 7275) & & & & & & & & & $<0.001$ \\
\hline Median and range & 34 & $7-100$ & 34 & $7-100$ & 36 & $13-90$ & 36 & 9-99 & \\
\hline Gender ( $n$ 7285) & & & & & & & & & 0.007 \\
\hline Men & 1121 & $15 \cdot 4$ & 908 & $14 \cdot 8$ & 121 & $17 \cdot 6$ & 92 & $19 \cdot 3$ & \\
\hline Women & 6164 & 84.6 & 5215 & $85 \cdot 2$ & 565 & 82.4 & 384 & $80 \cdot 7$ & \\
\hline Race/ethnicity & & & & & & & & & $<0.001$ \\
\hline Hispanic & 3612 & 49.5 & 3106 & $50 \cdot 7$ & 298 & $43 \cdot 1$ & 208 & 43.4 & \\
\hline White & 1316 & $18 \cdot 0$ & 1167 & $19 \cdot 1$ & 100 & 14.5 & 49 & $10 \cdot 2$ & \\
\hline Vietnamese & 787 & $10 \cdot 8$ & 596 & $9 \cdot 7$ & 118 & $17 \cdot 1$ & 73 & $15 \cdot 2$ & \\
\hline Other Asian & 758 & $10 \cdot 4$ & 593 & $9 \cdot 7$ & 87 & $12 \cdot 6$ & 78 & $16 \cdot 3$ & \\
\hline African American & 481 & $6 \cdot 6$ & 415 & $6 \cdot 8$ & 41 & 8.5 & 25 & $5 \cdot 2$ & \\
\hline East African & 212 & 2.9 & 128 & $2 \cdot 1$ & 41 & 5.9 & 43 & 9.0 & \\
\hline Multiple/Other & 132 & $1 \cdot 8$ & 122 & $2 \cdot 0$ & 7 & 1.0 & 3 & 0.6 & \\
\hline Survey language & & & & & & & & & $<0.001$ \\
\hline English & 4826 & $66 \cdot 1$ & 4134 & 67.5 & 458 & $66 \cdot 2$ & 234 & 48.9 & \\
\hline Spanish & 1827 & $25 \cdot 0$ & 1521 & $24 \cdot 8$ & 156 & $22 \cdot 5$ & 150 & $31 \cdot 3$ & \\
\hline Vietnamese & 470 & $6 \cdot 4$ & 347 & 5.7 & 57 & 8.2 & 66 & $13 \cdot 8$ & \\
\hline Chinese & 89 & $1 \cdot 2$ & 79 & 1.3 & 2 & 0.3 & 8 & $1 \cdot 7$ & \\
\hline Somali & 86 & $1 \cdot 2$ & 46 & 0.8 & 19 & $2 \cdot 8$ & 21 & $4 \cdot 4$ & \\
\hline Household members ( $n$ 7293) & & & & & & & & & 0.011 \\
\hline Median and range & $4 \cdot 0$ & $1-14$ & $4 \cdot 0$ & $1-14$ & $4 \cdot 0$ & $1-14$ & $4 \cdot 0$ & $1-11$ & \\
\hline Government assistance & & & & & & & & & $<0.001$ \\
\hline WIC & 4092 & $56 \cdot 1$ & 3571 & $58 \cdot 3$ & 324 & $46 \cdot 8$ & 197 & $41 \cdot 1$ & \\
\hline SNAP/CalFresh & 1958 & $26 \cdot 8$ & 1550 & $25 \cdot 3$ & 221 & 31.9 & 187 & $39 \cdot 0$ & \\
\hline SSI & 1248 & $17 \cdot 1$ & 1006 & $16 \cdot 4$ & 147 & $21 \cdot 2$ & 95 & $19 \cdot 8$ & \\
\hline Season of enrolment & & & & & & & & & $<0.001$ \\
\hline Spring & 1238 & $17 \cdot 0$ & 936 & $15 \cdot 3$ & 218 & 31.5 & 84 & $17 \cdot 5$ & \\
\hline Summer & 3839 & $52 \cdot 6$ & 3349 & $54 \cdot 7$ & 200 & 28.9 & 290 & $60 \cdot 5$ & \\
\hline Autumn & 1892 & $25 \cdot 9$ & 1643 & $26 \cdot 8$ & 145 & $21 \cdot 0$ & 104 & $21 \cdot 7$ & \\
\hline Winter & 329 & 4.5 & 199 & 3.3 & 129 & $18 \cdot 6$ & 1 & 0.2 & \\
\hline Enrolment market & & & & & & & & & $<0.001$ \\
\hline City Heights & 3112 & $42 \cdot 6$ & 2234 & $36 \cdot 5$ & 485 & $70 \cdot 1$ & 393 & $82 \cdot 1$ & \\
\hline Southeast San Diego & 232 & $3 \cdot 2$ & 222 & 3.6 & 10 & 1.5 & 0 & 0 & \\
\hline San Marcos & 2039 & $27 \cdot 9$ & 1780 & $29 \cdot 1$ & 173 & $25 \cdot 0$ & 86 & $18 \cdot 0$ & \\
\hline Golden Hill & 982 & 12.5 & 961 & $15 \cdot 7$ & 21 & 3.0 & 0 & 0 & \\
\hline Linda Vista & 933 & $12 \cdot 8$ & 930 & $15 \cdot 2$ & 3 & 0.4 & 0 & 0 & \\
\hline Number of visits & & & & & & & & & $<0.001$ \\
\hline Median and range & 1.0 & $1-36$ & 1.0 & $1-14$ & $6 \cdot 0$ & $2-24$ & $9 \cdot 0$ & $2-36$ & \\
\hline One & 3976 & 54.5 & 3976 & 64.9 & 0 & 0 & 0 & 0 & $<0.001$ \\
\hline Two & 1159 & $15 \cdot 9$ & 1013 & $16 \cdot 5$ & 115 & $16 \cdot 6$ & 31 & 6.5 & \\
\hline Three & 596 & $8 \cdot 2$ & 487 & 8.0 & 72 & $10 \cdot 4$ & 37 & $7 \cdot 7$ & \\
\hline Four & 358 & 4.9 & 258 & $4 \cdot 2$ & 71 & $10 \cdot 3$ & 23 & $3 \cdot 1$ & \\
\hline Five or more & 1209 & $16 \cdot 6$ & 393 & $6 \cdot 4$ & 434 & $62 \cdot 7$ & 382 & $79 \cdot 8$ & \\
\hline Total months of Fresh Fund use & & & & & & & & & \\
\hline Median and range & 0 & $0-20$ & 0 & $0-5 \cdot 9$ & $9 \cdot 0$ & $6-11.9$ & $15 \cdot 4$ & $12 \cdot 2-19 \cdot 5$ & \\
\hline Total money exchanged (\$US) & & & & & & & & & $<0.001$ \\
\hline Median and range & $20 \cdot 0$ & $1-711$ & 20 & $0-320$ & 100 & $6-560$ & 147 & $15-711$ & \\
\hline Servings $\mathrm{F} \& \mathrm{~V} / \mathrm{d} \ddagger(\mathrm{n}$ 6688) & & & & & & & & & $<0.001$ \\
\hline$<1$ & 167 & 2.5 & 125 & $2 \cdot 2$ & 22 & 3.5 & 20 & $4 \cdot 3$ & \\
\hline $1-2$ & 1819 & $27 \cdot 2$ & 1489 & $26 \cdot 7$ & 182 & $28 \cdot 7$ & 148 & 31.5 & \\
\hline $3-4$ & 3061 & $45 \cdot 8$ & 2557 & $45 \cdot 8$ & 283 & $44 \cdot 6$ & 221 & $47 \cdot 0$ & \\
\hline$\geq 5$ & 1614 & 24.5 & 1413 & $25 \cdot 3$ & 147 & 23.2 & 81 & $17 \cdot 2$ & \\
\hline Perceived diet quality $¥$ (n 6709) & & & & & & & & & $<0.001$ \\
\hline Very unhealthy & 326 & 4.9 & 191 & 3.4 & 57 & $9 \cdot 0$ & 78 & $16 \cdot 4$ & \\
\hline Unhealthy & 1171 & $17 \cdot 5$ & 717 & $12 \cdot 8$ & 200 & $31 \cdot 7$ & 254 & $53 \cdot 4$ & \\
\hline Average & 2384 & 35.5 & 2049 & $36 \cdot 6$ & 202 & $32 \cdot 0$ & 133 & $27 \cdot 9$ & \\
\hline Healthy & 2111 & 31.5 & 1972 & $35 \cdot 2$ & 130 & $20 \cdot 6$ & 9 & 1.9 & \\
\hline Very healthy & 717 & $10 \cdot 7$ & 673 & $12 \cdot 0$ & 42 & $6 \cdot 7$ & 2 & 0.4 & \\
\hline Weekly spending on F\&V $¥(n$ 6724) & & & & & & & & & $<0.001$ \\
\hline Less than \$US 10 & 258 & 3.8 & 203 & 3.6 & 24 & 3.8 & 31 & 6.5 & \\
\hline \$US 10-19 & 1555 & $23 \cdot 1$ & 1242 & $22 \cdot 1$ & 166 & $26 \cdot 1$ & 147 & $31 \cdot 0$ & \\
\hline \$US 20-\$29 & 1928 & 28.7 & 1556 & $22 \cdot 7$ & 211 & $33 \cdot 2$ & 161 & 33.9 & \\
\hline \$US 30-39 & 1173 & $17 \cdot 4$ & 996 & $17 \cdot 7$ & 106 & $16 \cdot 7$ & 71 & $6 \cdot 1$ & \\
\hline$\$$ US 40 or more & 1810 & $26 \cdot 9$ & 1616 & $28 \cdot 8$ & 129 & $20 \cdot 3$ & 65 & $13 \cdot 7$ & \\
\hline
\end{tabular}

WIC, Special Supplemental Nutrition Program for Women, Infants, and Children; SNAP, Supplemental Nutrition Assistance Program; SSI, Supplemental Security Income; F\&V, fruits and vegetables.

${ }^{*}$ Totals equal 7298 unless otherwise indicated.

$\dagger P$ values are based on $X^{2}$ tests or non-parametric Wilcoxon rank sum tests, and demonstrate overall significance of differences between total months of Fresh Fund use by each characteristic.

‡Self-reported during baseline survey. 
Table 2 Unadjusted Poisson regression analysis of the relationship of selected baseline characteristics with the number of Fresh Fund visits by total length of Fresh Fund use. San Diego, California, 2010-2012 ( $n$ 7298) ${ }^{\star}$

\begin{tabular}{|c|c|c|c|c|c|c|c|c|c|}
\hline \multirow[b]{2}{*}{ Characteristic } & \multicolumn{3}{|c|}{$\leq 6$ months $(n 6127)$} & \multicolumn{3}{|c|}{ 6-12 months ( $n$ 692) } & \multicolumn{3}{|c|}{$>12$ months $(n$ 479) } \\
\hline & $\mathrm{RR}$ & $95 \% \mathrm{Cl}$ & $P$ & $\mathrm{RR}$ & $95 \% \mathrm{Cl}$ & $P$ & $\mathrm{RR}$ & $95 \% \mathrm{Cl}$ & $P$ \\
\hline Age (10-year interval) ( $n$ 7275) & 1.07 & $1.06,1.08$ & $<0.001$ & 1.07 & $1.04,1.09$ & $<0.001$ & 1.03 & $0.99,1.07$ & 0.073 \\
\hline Men $(n 7285)$ & 1.21 & $1 \cdot 15,1 \cdot 28$ & $<0.001$ & 1.25 & $1.13,1.39$ & $<0.001$ & 1.20 & $1.05,1.37$ & 0.009 \\
\hline Race/ethnicity & & & $<0.001$ & & & $<0.001$ & & & 0.040 \\
\hline White & 1.00 & Ref. & & 1.00 & Ref. & & 1.00 & Ref. & \\
\hline Hispanic & 0.86 & $0.81,0.90$ & & 0.87 & $0.76,0.99$ & & $1 \cdot 12$ & $0.91,1.38$ & \\
\hline Vietnamese & 1.56 & $1.47,1.67$ & & 1.25 & $1.09,1.44$ & & 1.38 & $1.09,1.73$ & \\
\hline Other Asian & $1 \cdot 13$ & $1.07,1.23$ & & 1.37 & $1.18,1.58$ & & 1.28 & $1.02,1.61$ & \\
\hline African American & 0.95 & $0.87,1.04$ & & 0.93 & $0.76,1.15$ & & 1.04 & $0.76,1.44$ & \\
\hline East African & 1.18 & $1.04,1.34$ & & 1.06 & $0.87,1.23$ & & 1.21 & $0.93,1.57$ & \\
\hline Multiple/Other & 1.03 & $0.89,1.18$ & & 0.87 & $0.56,1.37$ & & 0.72 & $0.29,1.80$ & \\
\hline Survey language & & & $<0.001$ & & & $<0.001$ & & & 0.104 \\
\hline English & 1.00 & Ref. & & 1.00 & Ref. & & 1.00 & Ref. & \\
\hline Spanish & 0.87 & $0.84,0.92$ & & 0.80 & $0.74,0.86$ & & 1.06 & $0.99,1.13$ & \\
\hline Vietnamese & 1.58 & $1.48,1.69$ & & 1.24 & $1.12,1.37$ & & 1.22 & $1.13,1.32$ & \\
\hline Chinese & 0.91 & $0.77,1.09$ & & 1.56 & $1.01,2.43$ & & 0.87 & $0.68,1.10$ & \\
\hline Somali & $1 \cdot 10$ & $0.89,1.35$ & & 1.02 & $0.85,1.22$ & & 1.21 & $1.06,1.38$ & \\
\hline Household members ( $n$ 7293) & 0.98 & $0.97,0.99$ & $<0.001$ & 0.97 & $0.96,0.99$ & 0.036 & 1.00 & $0.97,1.03$ & 0.870 \\
\hline Government assistance & & & $<0.001$ & & & $<0.001$ & & & 0.003 \\
\hline WIC & 1.00 & Ref. & & 1.00 & Ref. & & 1.00 & Ref. & \\
\hline SNAP/CalFresh & 1.36 & $1.30,1.42$ & & 1.36 & $1.24,1.50$ & & $1 \cdot 16$ & $1.02,1.32$ & \\
\hline SSI & 1.51 & $1.44,1.59$ & & 1.45 & $1.31,1.61$ & & 1.28 & $1.10,1.49$ & \\
\hline Season of enrolment & & & $<0.001$ & & & $<0.001$ & & & 0.523 \\
\hline Summer & 1.00 & Ref. & & 1.00 & Ref. & & 1.00 & Ref. & \\
\hline Autumn & 0.88 & $0.84,0.92$ & & 1.24 & $1.09,1.40$ & & 0.92 & $0.80,1.06$ & \\
\hline Winter & 0.97 & $0.87,1.09$ & & 1.49 & $1.32,1.40$ & & 1.05 & $0.90,1.21$ & \\
\hline Spring & 0.92 & $0.87,0.98$ & & 1.33 & $1.19,1.49$ & & 1.05 & $0.90,1.21$ & \\
\hline Enrolment market & & & $<0.001$ & & & $<0.001$ & & & $<0.001$ \\
\hline City Heights & 1.00 & Ref. & & 1.00 & Ref. & & 1.00 & Ref. & \\
\hline Southeast San Diego & 0.67 & $0.59,0.75$ & & 0.93 & $0.72,1.19$ & & $-\dagger$ & - & \\
\hline San Marcos & 0.73 & $0.69,0.76$ & & 0.71 & $0.65,0.76$ & & 0.66 & $0.61,0.72$ & \\
\hline Golden Hill & 0.70 & $0.66,0.74$ & & 0.88 & $0.73,1.05$ & & $-\dagger$ & - & \\
\hline Linda Vista & 1.26 & $1 \cdot 19,1.32$ & & 0.79 & $0.48,1.28$ & & $-\dagger$ & - & \\
\hline Servings F\&V/d ( $n$ 6688) & & & 0.060 & & & 0.062 & & & 0.828 \\
\hline$<1$ & 1.00 & Ref. & & 1.00 & Ref. & & 1.00 & Ref. & \\
\hline $1-2$ & 1.23 & $1.05,1.43$ & & 0.78 & $0.63,0.98$ & & 1.03 & $0.77,1.39$ & \\
\hline $3-4$ & 1.21 & $1.04,1.41$ & & 0.74 & $0.59,0.92$ & & 1.09 & $0.81,1.46$ & \\
\hline$\geq 5$ & 1.22 & $1.05,1.42$ & & 0.74 & $0.59,0.94$ & & 1.09 & $0.80,1.48$ & \\
\hline Perceived diet quality ( $n$ 6708) & & & $<0.001$ & & & 0.275 & & & 0.025 \\
\hline Very unhealthy & 1.00 & Ref. & & 1.00 & Ref. & & 1.00 & Ref. & \\
\hline Unhealthy & 0.85 & $0.76,0.96$ & & 1.05 & $0.88,1.24$ & & 1.02 & $0.87,1.18$ & \\
\hline Average & 0.88 & $0.79,0.98$ & & 0.99 & $0.83,1.19$ & & 0.82 & $0.69,0.97$ & \\
\hline Healthy & 0.96 & $0.86,1.07$ & & 1.14 & $0.95,1.36$ & & 0.89 & $0.58,1.38$ & \\
\hline Very healthy & 1.00 & $0.89,1.13$ & & $1 \cdot 12$ & $0.88,1.41$ & & $1 \cdot 15$ & $0.53,2.53$ & \\
\hline Weekly spending on F\&V ( $n$ 6728) & & & 0.262 & & & $<0.001$ & & & $<0.001$ \\
\hline \$US 40 or more & 1.00 & Ref. & & 1.00 & Ref. & & 1.00 & Ref. & \\
\hline$\$$ US 30-39 & 0.95 & $0.89,1.01$ & & 0.99 & $0.89,1 \cdot 10$ & & 0.98 & $0.88,1 \cdot 10$ & \\
\hline \$US 20-29 & 1.02 & $0.96,1.07$ & & 1.03 & $0.94,1.13$ & & $1 \cdot 10$ & $1.01,1.21$ & \\
\hline \$US $10-\$ 19$ & 0.99 & $0.94,1.05$ & & 1.08 & $0.98,1.75$ & & $1 \cdot 16$ & $1.05,1.27$ & \\
\hline Less than \$US 10 & 0.98 & $0.88,1.10$ & & 1.46 & $1.25,1.70$ & & 1.23 & $1.08,1.40$ & \\
\hline
\end{tabular}

RR, relative risk; WIC, Special Supplemental Nutrition Program for Women, Infants, and Children; SNAP, Supplemental Nutrition Assistance Program; SSI, Supplemental Security Income; F\&V, fruits and vegetables; ref., reference category.

${ }^{\star}$ Totals equal 7298 unless otherwise indicated. tn 0 .

survey; and among 582 individuals who returned multiple times for at least 12 months, 252 completed a baseline and a follow-up survey.

Tables 2 and 3 display the results of bivariate and multivariate Poisson regression analyses stratified by total months of Fresh Fund use, respectively. Among those who came to Fresh Fund for $\leq 6$ months, the unadjusted models showed all variables except weekly spending on F\&V at baseline to be statistically significant for number of Fresh
Fund visits. All tolerance values were above $0 \cdot 10$, therefore we concluded that multicollinearity did not impact the models. Multivariate Poisson regression showed that individuals identifying as Hispanic ethnicity ( $v$. Whites; relative risk $(\mathrm{RR})=0.92 ; 95 \% \mathrm{CI} 0.87,0.97)$, enrolling in the autumn $(v$. summer; RR $=0.77 ; 95 \%$ CI $0.74,0.81)$ and attending the Southeast San Diego (RR $=0.71 ; 95 \% \mathrm{CI}$ $0.63,0.80)$, San Marcos (RR $=0.78 ; 95 \%$ CI $0.74,0.82$ ) or Golden Hill (RR $=0.77 ; 95 \%$ CI $0.72,0.82)$ market $(v$. City 
Table 3 Multivariate Poisson regression analysis* of the relationship of selected baseline characteristics with the number of Fresh Fund visits by total length of Fresh Fund use. San Diego, California, 2010-2012 ( $n$ 7298)†

\begin{tabular}{|c|c|c|c|c|c|c|c|c|c|}
\hline & \multicolumn{3}{|c|}{$\leq 6$ months $(n 5134)$} & \multicolumn{3}{|c|}{ 6-12 months ( $n$ 625) } & \multicolumn{3}{|c|}{$>12$ months ( $n$ 468) } \\
\hline & $\mathrm{RR}$ & $95 \% \mathrm{Cl}$ & $P$ & $\mathrm{RR}$ & $95 \% \mathrm{Cl}$ & $P$ & $\mathrm{RR}$ & $95 \% \mathrm{Cl}$ & $P$ \\
\hline Age (10-year interval) & 0.98 & $0.99,1.00$ & 0.176 & $-\ddagger$ & - & - & 0.96 & $0.91,1.01$ & 0.128 \\
\hline Men & - & - & - & $\stackrel{+}{-}$ & - & - & $1 \cdot 16$ & $1.00,1.35$ & 0.052 \\
\hline Race/ethnicity & & & $<0.001$ & & & $<0.001$ & & & - \\
\hline White & 1.00 & Ref. & & 1.00 & Ref. & & - & & \\
\hline Hispanic & 0.92 & $0.87,0.97$ & & 0.91 & $0.80,1.04$ & & - & - & \\
\hline Vietnamese & $1 \cdot 18$ & $1.07,1.30$ & & $1 \cdot 13$ & $0.95,1.35$ & & - & - & \\
\hline Other Asian & 1.11 & $1.03,1.20$ & & 1.23 & $1.04,1.46$ & & - & - & \\
\hline African American & 0.94 & $0.86,1.02$ & & 0.80 & $0.64,0.99$ & & - & - & \\
\hline East African & 1.01 & $0.88,1.17$ & & 0.89 & $0.72,1.10$ & & - & - & \\
\hline Multiple/Other & 1.00 & $0.88,1 \cdot 13$ & & 0.84 & $0.53,1.33$ & & - & - & \\
\hline Survey language & & & 0.173 & & & - & & & - \\
\hline English & 1.00 & Ref. & & - & & & - & & \\
\hline Spanish & 1.00 & $0.95,1.06$ & & - & - & & - & - & \\
\hline Vietnamese & 1.04 & $0.94,1.15$ & & - & - & & - & - & \\
\hline Chinese & 0.70 & $0.52,0.95$ & & - & - & & - & - & \\
\hline Somali & 0.99 & $0.80,1.25$ & & - & - & & - & - & \\
\hline Government assistance & & & $<0.001$ & & & $<0.001$ & & & 0.081 \\
\hline WIC & 1.00 & Ref. & & 1.00 & Ref. & & 1.00 & Ref. & \\
\hline SNAP/CalFresh & 1.26 & $1.20,1.32$ & & 1.22 & $1.09,1.35$ & & 1.04 & $0.92,1.20$ & \\
\hline SSI & 1.29 & $1 \cdot 19,1.32$ & & $1 \cdot 10$ & $0.95,1.28$ & & 1.30 & $1.03,1.63$ & \\
\hline Season of enrolment & & & $<0.001$ & & & $<0.001$ & & & - \\
\hline Summer & 1.00 & Ref. & & 1.00 & Ref. & & - & - & \\
\hline Autumn & 0.77 & $0.74,0.81$ & & 1.14 & $1.00,1.30$ & & - & - & \\
\hline Winter & 0.93 & $0.84,1.03$ & & 1.29 & $1.13,1.48$ & & - & - & \\
\hline Spring & 1.01 & $0.95,1.06$ & & 1.23 & $1.08,1.38$ & & - & - & \\
\hline Enrolment market & & & $<0.001$ & & & 0.027 & & & $<0.001$ \\
\hline City Heights & 1.00 & Ref. & & 1.00 & & Ref. & 1.00 & Ref. & \\
\hline Southeast San Diego & 0.71 & $0.63,0.80$ & & 0.94 & $0.67,1.32$ & & $-\S$ & - & \\
\hline San Marcos & 0.78 & $0.74,0.82$ & & 0.82 & $0.73,0.93$ & & 0.68 & $0.57,0.80$ & \\
\hline Golden Hill & 0.77 & $0.72,0.82$ & & 0.98 & $0.76,1.27$ & & $-\S$ & - & \\
\hline Linda Vista & 1.19 & $1.13,1.26$ & & $1 \cdot 10$ & $0.51,2.37$ & & $-\S$ & - & \\
\hline Servings $F \& V / d$ & & & 0.012 & & & - & & & - \\
\hline$<1$ & 1.00 & Ref. & & - & - & & - & - & \\
\hline $1-2$ & 1.24 & $1.08,1.43$ & & - & - & & - & - & \\
\hline $3-4$ & 1.24 & $1.09,1.43$ & & - & - & & - & - & \\
\hline$\geq 5$ & 1.26 & $1.09,1.44$ & & - & - & & - & - & \\
\hline Perceived diet quality & & & 0.050 & & & - & & & 0.060 \\
\hline Very unhealthy & 1.00 & Ref. & & - & - & & 1.00 & Ref. & \\
\hline Unhealthy & 0.90 & $0.81,1.00$ & & - & - & & 1.05 & $0.90,1.22$ & \\
\hline Average & 0.92 & $0.83,1.02$ & & - & - & & 0.87 & $0.73,1.03$ & \\
\hline Healthy & 0.94 & $0.86,1.04$ & & - & - & & 1.12 & $0.73,1.74$ & \\
\hline Very healthy & 0.98 & $0.88,1.10$ & & & & & $1 \cdot 29$ & $0.46,3.60$ & \\
\hline
\end{tabular}

RR, relative risk; WIC, Special Supplemental Nutrition Program for Women, Infants, and Children; SNAP, Supplemental Nutrition Assistance Program; SSI, Supplemental Security Income; F\&V, fruits and vegetables; ref., reference category.

${ }^{*}$ Adjusted for all other variables in the multivariate model.

†Totals equal 7298 unless otherwise indicated.

$\ddagger$ Dashes indicate variables that were not included in the multivariate model. $\S n 0$.

Heights) had fewer visits after controlling for all other characteristics. More visits to the market were independently associated with identifying as Vietnamese ( $\mathrm{RR}=$ $1.18 ; 95 \%$ CI $1.07,1.30$ ) and other Asian race/ethnicity $(\mathrm{RR}=1 \cdot 11 ; 95 \% \mathrm{CI} 1.03,1 \cdot 20) ; \mathrm{SNAP}(\mathrm{RR}=1 \cdot 26 ; 95 \% \mathrm{CI}$ $1 \cdot 20,1.32)$ and SSI (RR $=1.29$; $95 \%$ CI $1.19,1 \cdot 32)$ assistance $(v$. WIC); attending the Linda Vista $(\mathrm{RR}=1 \cdot 19 ; 95 \%$ CI $1.13,1.26)$ market ( $v$. City Heights); and baseline reporting of $\mathrm{F} \& \mathrm{~V}$ consumption of $1-2$ servings/d (RR $=$ 1.24; $95 \%$ CI 1.08, 1.43), 3-4 servings/d ( $R R=1.24 ; 95 \%$ CI $1.09,1.43)$ and $\geq 5$ servings/d (RR $=1.26 ; 95 \%$ CI 1.09 , 1.44) (v. $<2$ servings F\&V/d). Baseline perception of diet quality as 'very unhealthy' was marginally associated
$(P=0.05)$ with more Fresh Fund visits compared with healthier perceptions of diet quality. Among those who came to Fresh Fund for 6-12 months, controlling for all other variables in the model, fewer visits was associated with identifying as African American (RR $=0.80 ; 95 \% \mathrm{CI}$ $0.64,0.99)$ and attending the San Marcos market $(\mathrm{RR}=0.82 ; 95 \% \mathrm{CI} 0.73,0.93)$; and a greater number of visits was associated with identifying as other Asian race/ ethnicity ( $R R=1.23 ; 95 \%$ CI 1.04, 1.46), SNAP assistance $(\mathrm{RR}=1 \cdot 22 ; 95 \% \mathrm{CI} 1 \cdot 09,1 \cdot 35)$, and winter $(\mathrm{RR}=1 \cdot 29 ; 95 \%$ CI $1.13,1.48)$ and spring $(\mathrm{RR}=1.23 ; 95 \%$ CI $1.08,1.38)$ enrolment. Among those who came to the markets for $>12$ months, controlling for all other variables, SSI 
Table 4 Linear regression analysis of the relationship of selected baseline characteristics with government assistance and personal money exchanged by length of Fresh Fund use. San Diego, California, 2010-2012 (n 7298)

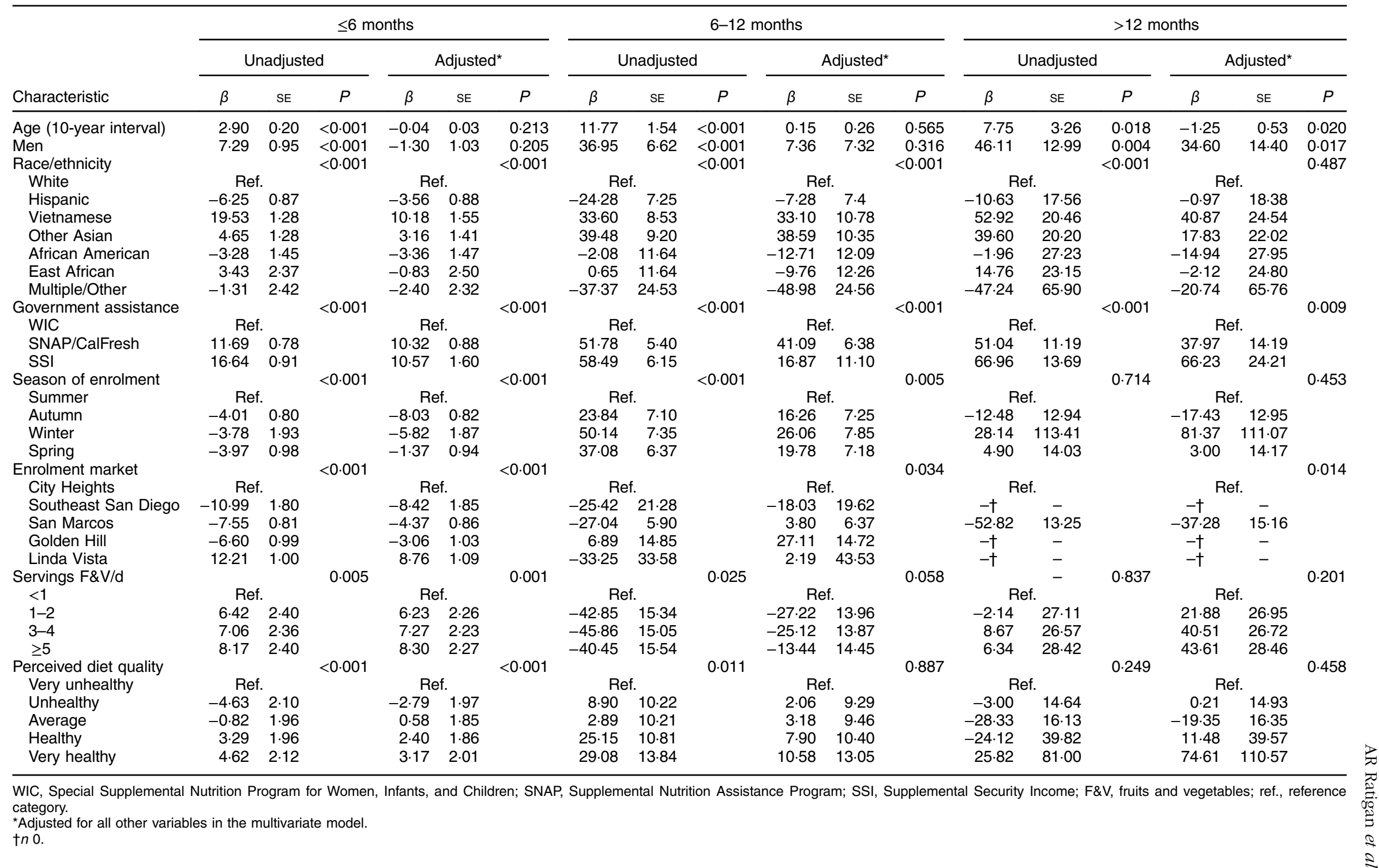


assistance continued to be associated with a greater number of visits ( $R R=1.30 ; 95 \%$ CI 1.03, 1.63). The only other variable marginally associated with number of visits for these $>12$ month users was male gender $(R R=1 \cdot 16$; $95 \%$ CI 1.00, 1.35). In linear regression analyses, after adjustment, those who reported consuming a greater number of servings of $\mathrm{F} \& \mathrm{~V}$ daily $(v,<1$ serving/d) and those who reported a healthy or very healthy diet ( $v$. a very unhealthy diet) at baseline exchanged significantly more money, but only among those who came to Fresh Fund for 6 months or less. These associations were not significant for the groups who came longer than 6 months (Table 4).

Finally, the results from the mixed-effects modelling showed, on average, that the total amount of money exchanged increased by \$US $0 \cdot 12$ per month of Fresh Fund use $(P<0 \cdot 001)$, that the within-individual odds of an increasing number of servings of $F \& V$ consumed increased by $2 \%$ per month of Fresh Fund use (OR $=1.02$; $95 \%$ CI $1.01,1.03 ; P=0.003$ ), and that the odds of improved perception of diet quality increased by $10 \%$ per month of Fresh Fund use (OR $=1.10$; CI 1.09, 1.11; $P<0 \cdot 001$; data not shown)

\section{Discussion}

Our analysis of this policy, systems and environmental intervention contributes to the evidence that farmers' market monetary incentive programmes may improve affordability and access to fresh F\&V among low-income individuals and families. Over 7000 government nutrition assistance recipients enrolled in Fresh Fund during the evaluation period, with significant increases in selfreported $F \& V$ consumption, improvement in the perception of overall diet quality and increased spending of personal money and government assistance money seen with continued use of Fresh Fund. These findings are consistent with previous US studies in which increased spending on and consumption of F\&V were found to be associated with the use of incentive programmes among SNAP and WIC users ${ }^{(26,27,29-32)}$. Although other similar studies have found positive associations between incentive programme use and consumption of $F \& V$, the present study is the first of its kind to find increased consumption with continued market use among low-income consumers using monetary incentives. Furthermore, upon the examination of independent predictors of repeated Fresh Fund use, participants who reported unhealthier diets at baseline were found to be marginally more likely to return to Fresh Fund a greater number of times than those who reported a healthier diet, but only among short-term users (6 months or less), suggesting that those who regarded their diets as unhealthier at baseline may have been initially more eager to improve their diet quality; however, over time they may have realized it was not sustainable for a variety of possible reasons (e.g. barriers to access or lack of variety of food items) and therefore did not continue to use the programme past 6 months.

Seasonal and market differences were significantly associated with continued use of Fresh Fund. Among those who came to the market only for 6 months, those who enrolled in the autumn compared with the summer were less likely to have multiple visits; however, among those who stayed for 6 to 12 months, winter and spring enrolment were both associated with more visits than summer enrolment. This could be because those who enrolled in winter and spring had more reason or desire to return during the spring and summer months when there was likely to be a wider variety of produce available at the markets; whereas those who enrolled in the summer would have less time before the autumn and winter months when variety may have been more limited. Clearly the City Heights and Linda Vista markets were more likely to have repeat visitors than the other Fresh Fund markets. These were also the most established of the markets in their neighbourhoods; in fact, both markets had functioning community advisory committees. Interestingly, the Linda Vista neighbourhood also had a large Asian population, which may have influenced these results. It is recommended that future research be conducted to examine roles of market characteristics on continued use of farmers' markets. The one factor most highly associated with number of visits for short-, medium- and long-term participants was their use of government assistance money in the form of SNAP or SSI. SSI-participating patrons who stayed for over 12 months were 1.3 times more likely to have a greater number of visits. Many SSI participants may be elderly or disabled and thus may have been more likely to continue using the market for food resources longer than the generally younger WIC participants who were of childbearing age. It was interesting that the three Poisson regression models for short-, medium- and long-term users demonstrated different results, with more variables (including baseline very unhealthy diets) being associated with number of visits for short-term users than long-term users. The number of visits for shorter-term participants was related to ethnicity, type of government assistance, enrolment market, season of enrolment, baseline F\&V servings/d and perceived diet quality. Longer participation was predominantly associated with type of income. SSI recipients who used the market for 12 months or more were the most likely to have the greatest number of visits.

Limitations must be kept in mind when interpreting these results. Because Fresh Fund was a policy, systems and environmental intervention, it was not meant to manipulate individuals' behaviours, and the data available for the current analysis were limited to the information collected among a convenience sample of those who voluntarily chose to participate in the baseline and followup surveys. In addition, perception of overall diet quality, daily consumption of F\&V and weekly spending on F\&V 
were self-reported and therefore may introduce reporting bias into the data. The generalizability of the results to populations in other geographic regions may be limited. However, our sample consisted of WIC, SNAP and SSI recipients with a diverse make-up of sexes ( $15 \%$ male), ages ( 7 to 100 years) and various racial/ethnic groups, which may improve the external validity of these results as they might apply to government nutrition assistance recipients in other urban locations. Fresh Fund was conducted in San Diego where weather likely plays a role in the availability of and attendance at farmers' markets throughout the year, whereas markets in colder climates likely close during the winter months. However, evaluations of farmers' market incentive programmes taking place in cities with significant winter weather (Philadelphia (Philly Food Bucks) and New York (Health Bucks)) have found similar results, with increased spending on and self-reported consumption of F\&V among SNAP and WIC participants ${ }^{(26,27,30)}$. Since this was not a behavioural intervention, the design did not include plans to actively retain participants, but rather to examine participation patterns over time. Participants continued to visit the market based on their own perceptions of need and over half of participants (55\%) visited the market once only. However, Dimitri and colleagues had a similar retention rate (49\%) in their longitudinal pilot study among SNAP and WIC shoppers ${ }^{(33)}$. It is unknown whether participants continued to shop at the farmers' markets if they chose not to obtain Fresh Fund incentive tokens, in which case they were not required to report to the Fresh Fund booth; however, this is unlikely. Despite these limitations, the present study has multiple strengths. There was a large sample comprised of racially diverse groups, a wide range of ages and a reasonable proportion of male participants. Additionally, the analysis incorporated longitudinal measures, adding to the current cross-sectional evidence surrounding farmers' market incentive programmes.

Given the robust health benefits of diets rich in F\&V and the evidence that the general US population does not consume nearly enough servings of F\&V, farmers' market incentive programmes have the potential to affect the health of low-income populations. Results showed that SSI government funding remained the factor most associated with number of visits to the market among those who remained for 12 months or more. In addition, the total amount of money spent at a Fresh Fund market and the self-reported number of servings of $F \& V$ consumed daily increased monthly with the length of participation. Furthermore, the perception of diet quality increased over time, highlighting a potential health benefit of improving and sustaining access to, and affordability of, farmers' markets among economically disadvantaged populations. The results of the present study can be used to inform future policy and practice interventions within the USA (e.g. policy, systems and environmental interventions). While the data from the present study have specific geographic and demographic context, they contribute to the evidence already provided by other incentive programmes in the USA (i.e. Philly Food Bucks and New York Health Bucks), which both showed promise in sustaining farmers' market attendance and F\&V consumption of low-income consumers. Statistics from other countries indicate that farmers' markets are not as widespread as they are in the $\mathrm{USA}^{(16-19)}$; and to our knowledge, none have implemented incentive or voucher programmes for low-income populations. However, these results can contribute to developing a framework for designing and implementing similar programmes in countries where low-income populations could benefit from farmers' market incentive programmes. Among countries where F\&V consumption has been marginalized by the Western, high-energy and low-nutrient diet, increasing and sustaining Fresh Fund-type programme operations and utilization, by those populations most at need, may stand to provide more long-term healthy behaviour and in turn health benefits.

\section{Acknowledgements}

Financial support: The project was supported in part by a cooperative agreement with the CDC (grant number 1 U58DP002496-01). Portions of this project's work involve the Communities Putting Prevention to Work (CPPW) Initiative supported by CDC funding. The CDC had no role in the design analysis, or writing of this article. Conflict of interest: None. Authorship: A.R.R. conducted the analysis and led the manuscript writing; S.L. was principal investigator on the evaluation and supervised the design, analysis and writing of the current study; H.L. assisted with the design and implementation of the analysis, interpretation of results and contributed to manuscript writing; C.D.C., C.A.M.A., T.A.C. and D.K.B. assisted with design, analysis and writing of the manuscript; W.J.W., the Director of Public Health Services at the County of San Diego Health and Human Services Agency, oversaw all aspects of study design and implementation and contributed to manuscript writing. Ethics of buman subject participation: This practice-based evaluation was conducted by an academiccommunity partnership according to the guidelines laid down in the Declaration of Helsinki and all procedures involving human subjects/patients were approved by the San Diego State University Institutional Review Board. Additional approval for the present study was obtained from the University of California San Diego Human Research Protection Program. Written informed consent was obtained from all participants.

\section{References}

1. Marshall TA (2011) Dietary Guidelines for Americans, 2010: an update. J Am Dent Assoc 142, 654-656.

2. Centers for Disease Control and Prevention (2010) State-specific trends in fruit and vegetable consumption 
among adults - United States, 2000-2009. MMWR Morb Mortal Wkly Rep 59, 1125-1130.

3. Statistics Canada (2015) Health Fact Sheets: Fruit and Vegetable Consumption, 2014. http://www.statcan.gc.ca/pub/82625-x/2015001/article/14182-eng.htm (accessed March 2017).

4. Roberts C (2015) Chapter 7: Fruit and vegetable consumption. In Health Survey for England 2013. vol. 1 [R Craig and J Mindell, editors]. http://content.digital.nhs.uk/catalogue/ PUB16076/HSE2013-Ch7-fru-veg-com.pdf (accessed March 2017).

5. Australian Bureau of Statistics (2013) Daily intake of fruits and vegetables. In Australian Health Survey: Updated Results, 2011-2012. Catalogue no. 4364.0.55.003. http://www.abs.gov. au/ausstats/abs@.nsf/Lookup/C549D4433F6B74D7CA257B8 200179569?opendocument (accessed March 2017).

6. Hall JN, Moore S, Harper SB et al. (2009) Global variability in fruit and vegetable consumption. Am J Prev Med $\mathbf{3 6}$, 402-409.

7. Dubowitz T, Heron M, Bird CE et al. (2008) Neighborhood socioeconomic status and fruit and vegetable intake among whites, blacks, and Mexican Americans in the United States. Am J Clin Nutr 87, 1883-1891.

8. Centers for Disease Control and Prevention (2007) Fruit and vegetable consumption among adults - United States, 2005. MMWR Morb Mortal Wkly Rep 56, 213-217.

9. Grimm KA, Foltz JL, Blanck HM et al. (2012) Household income disparities in fruit and vegetable consumption by state and territory: results of the 2009 Behavioral Risk Factor Surveillance System. J Acad Nutr Diet 112, 2014-2021.

10. Moore LV, Roux AVD, Nettleton JA et al. (2008) Associations of the local food environment with diet quality - a comparison of assessments based on surveys and geographic information systems. Am J Epidemiol 167, 917-924.

11. Moore LV \& Roux AVD (2006) Associations of neighborhood characteristics with the location and type of food stores. Am J Public Health 96, 325-331.

12. Rose D \& Richards R (2004) Food store access and household fruit and vegetable use among participants in the US Food Stamp Program. Public Health Nutr 7, 1081-1088.

13. Gustafson A, Lewis S, Perkins S et al. (2013) Neighbourhood and consumer food environment is associated with dietary intake among Supplemental Nutrition Assistance Program (SNAP) participants in Fayette County, Kentucky. Public Health Nutr 16, 1229-1237.

14. Baker EA, Schootman M, Barnidge E et al. (2006) The role of race and poverty in access to foods that enable individuals to adhere to dietary guidelines. Prev Chronic Dis 3, A76.

15. Powell LM, Slater S, Mirtcheva D et al. (2007) Food store availability and neighborhood characteristics in the United States. Prev Med 44, 189-195.

16. US Department of Agriculture (2017) Local Food Directories: National Farmers Market Directory. https://www.ams.usda. gov/local-food-directories/farmersmarkets (accessed March 2017).

17. Australia Farmers' Markets Association (2017) Markets Directory. http://farmersmarkets.org.au/find-a-market/ (accessed March 2017).

18. Department for Environment, Food \& Rural Affairs (2013) Farming Business: Farm shops and farmers' markets. https://www.gov.uk/guidance/farm-shops-and-farmersmarkets (accessed March 2017).

19. Farmers Market Canada (2009) National Farmers' Market Impact Study 2009 Report. http://www.unbc.ca/sites/default/ files/sections/david-connell/farmers-markets/nationalfarmers marketimpactstudy2009.pdf (accessed March 2017).
20. US Department of Agriculture (2015) Comparison of SNAP Authorized Farmers and Markets FY2008 and FY2015. https://origin.drupal.fns.usda.gov/sites/default/files/snap/ SNAP-FM-093015.pdf (accessed July 2017).

21. US Department of Agriculture (2017) WIC Farmers' Market Nutrition Program (FMNP). http://www.fns.usda.gov/fmnp/ wic-farmers-market-nutrition-program-fmnp (accessed July 2017).

22. US Department of Agriculture (2017) SNAP and Farmers Markets. http://www.fns.usda.gov/ebt/learn-about-snapbenefits-farmers-markets (accessed July 2017).

23. Jilcott Pitts SB, Wu Q, Demarest CL et al. (2015) Farmers' market shopping and dietary behaviours among Supplemental Nutrition Assistance Program participants. Public Health Nutr 18, 2407-2414.

24. Wheeler AL \& Chapman-Novakofski K (2014) Farmers' markets: costs compared with supermarkets, use among WIC clients, and relationship to fruit and vegetable intake and related psychosocial variables. J Nutr Educ Behav 46, 3 Suppl., S65-S70.

25. Grin BM, Gayle TL, Saravia DC et al. (2013) Use of farmers markets by mothers of WIC recipients, Miami-Dade County, Florida, 2011. Prev Chronic Dis 10, E95.

26. Baronberg S, Dunn L, Nonas C et al. (2013) The impact of New York City's Health Bucks Program on electronic benefit transfer spending at farmers markets, 2006-2009. Prev Chronic Dis 10, E163.

27. Olsho LE, Payne GH, Walker DK et al. (2015) Impacts of a farmers' market incentive programme on fruit and vegetable access, purchase and consumption. Public Health Nutr 18, 2712-2721.

28. Lindsay S, Lambert J, Penn T et al. (2013) Monetary matched incentives to encourage the purchase of fresh fruits and vegetables at farmers markets in underserved communities. Prev Chronic Dis 10, E188.

29. Freedman DA, Mattison-Faye A, Alia K et al. (2014) Comparing farmers' market revenue trends before and after the implementation of a monetary incentive for recipients of food assistance. Prev Chronic Dis 11, E87.

30. Young CR, Aquilante JL, Solomon S et al. (2013) Improving fruit and vegetable consumption among low-income customers at farmers markets: Philly Food Bucks, Philadelphia, Pennsylvania, 2011. Prev Chronic Dis 10, E166.

31. Herman DR, Harrison GG, Afifi AA et al. (2008) Effect of a targeted subsidy on intake of fruits and vegetables among low-income women in the Special Supplemental Nutrition Program for Women, Infants, and Children. Am J Public Health 98, 98-105.

32. Racine EF, Vaughn AS \& Laditka SB (2010) Farmers' market use among African-American women participating in the Special Supplemental Nutrition Program for Women, Infants, and Children. J Am Diet Assoc 110, 441-446.

33. Dimitri C, Oberholtzer L, Zive M et al. (2015) Enhancing food security of low-income consumers: an investigation of financial incentives for use at farmers markets. Food Policy 52, 64-70.

34. Nichols P, Ussery-Hall A, Griffin-Blake S et al. (2012) The evolution of the steps program, 2003-2010: transforming the federal public health practice of chronic disease prevention. Prev Chronic Dis 9, E50.

35. Bunnell R, O'Neil D, Soler R et al. (2012) Fifty communities putting prevention to work: accelerating chronic disease prevention through policy, systems and environmental change. J Community Health 37, 1081-1090. 